En effet, il y a pour les perceptions de cette nature une cerlaine incertitude dans la pratique administrative. Les redevances pour occupations de cette nature, étant comme toute redevance un droit appartenant au propriétaire du sol, devraient revenir aux communes dont la route de grande communication traverse successivement les territorres.

D'autre part, la police de la route appartenant à l'Etat, 1l se peut que ce sort l'Etat qui perçoive plus ou moins régulièrement les dites redevances. Selon le cas, c'est-à-dire selon que la réclamation sera faite par l'agent de l'Etat, ou par l'agent de la commune, on écrira à l'un ou à l'autre pour lu1 faire l'offre des droits d'après les nouveaux tarifs.

Paul Bougault, Arocal à la Co.ll d'tippel de Lyon.

\section{LE RENFORCEUR DE CHUTE}

L'éminent hydraulicien auquel nous devons le compteur venturi, M. Clemens Herschel, a publié récemment dans le Harvard Engineering Journal (juin 1908) une très intéressante étude sur l'appareil qu'il a nommé Renforceur de chute (Fall-Increaser), et qu'il a éprouvé au Bief Tareur de Holyoke (*).

Cet appareil est essentiellement constitué par deux troncs de cône, l'un convergent, l'autre divergent, réunis par leur sommet commun. Il a la forme d'un compteur Venturı dont la grorge aurait disparu, mais la dépression,

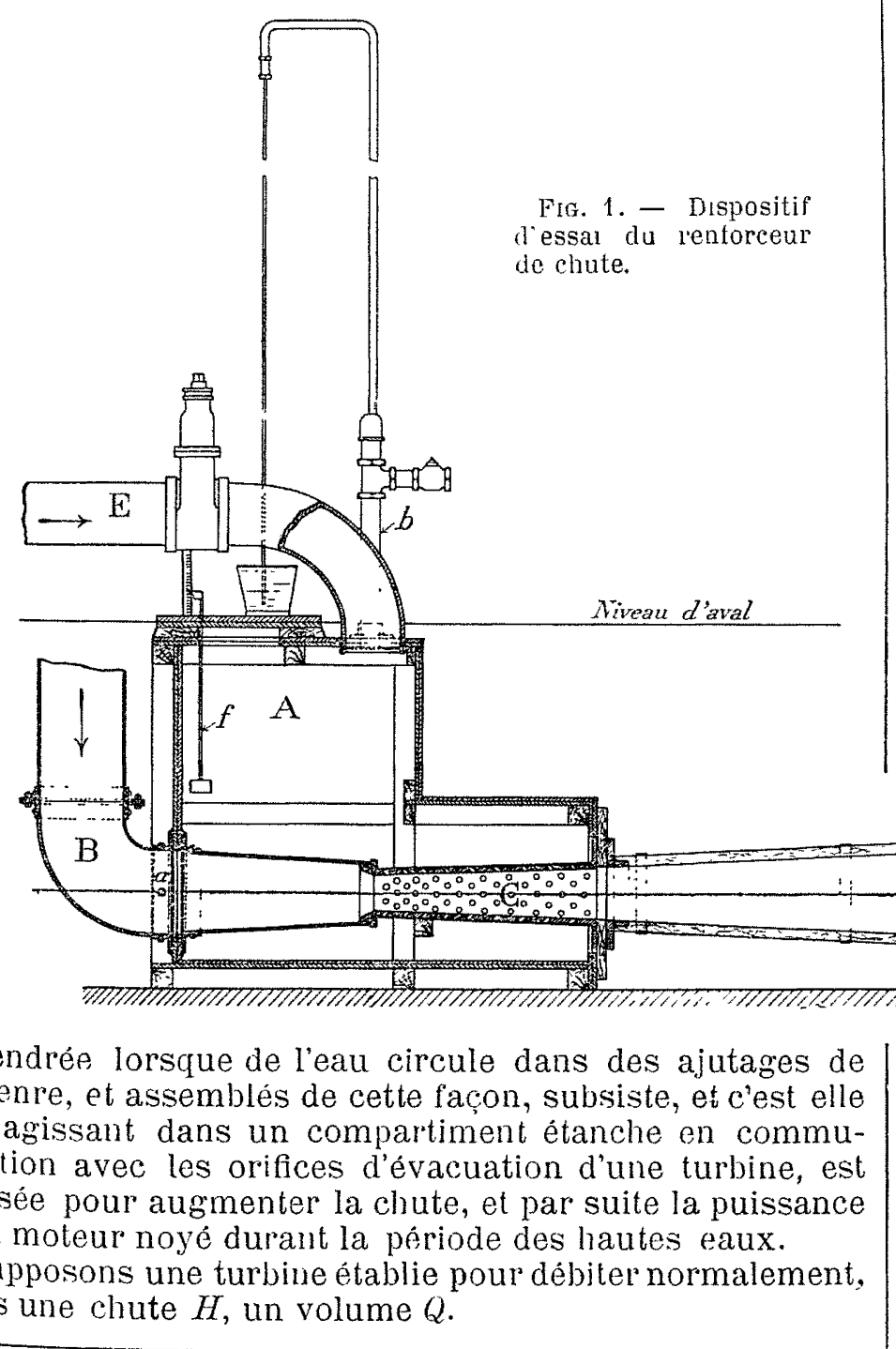

(') Ja description de ce Bief tareur a blé donnée par M, Blanchst dans la Houille Blanche de janvier 1904.
En désignant par os l'aire totale donnant dans la turbine effectivement passage à leau dans les conditions supposées, et par $P$ la puissance de cette turbine, nous aurons théoriquernent :

$$
Q=m \omega / \overline{2 g H} \quad P=Q H=m \omega / \overline{2 g} H^{\frac{3}{2}}
$$

En hautes eaux, la chute est réduite à $h$. Le volume débité et la puissance deviendront alor:s:

$$
q=n \omega \sqrt{2 g h} \quad p=q h=m \omega V \overline{2 g} h^{\frac{3}{2}}
$$

Si l'on met les orifices de sortie d: la turbine en communication avec un milieu dans lequel existe une dépression, celle-ci peut être représentée par une charge additionnelle $h$ ' qui s'ajoutera à $h$, et alors on écrira, $q$ ' et $l$ ' étant le nouveau débit et la nouvelle puissance du moteur :

$$
\begin{gathered}
q^{\prime}=m \omega \sqrt{2 g(h+h)} \\
p^{\prime}=q^{\prime}(h+h)=m \omega / \overline{2 g}\left[h+h^{\prime}\right]^{\frac{3}{2}}
\end{gathered}
$$

L'accroissement de puissance sera :

d'ailleurs :

$$
\Delta P=q^{\prime}(h+h)-q h
$$

d'où :

$$
\frac{q}{q}=\frac{V \bar{h}}{V \overline{h+h}}
$$

$$
\Delta P=q\left[\frac{\left(h+h h^{\frac{2}{2}}-h^{\frac{1}{2}}\right.}{V h+h}\right]
$$

Cet accros:sement de puissance est obtenu en envoyant dans le renforceur, sous une charge $h$, un volume d'eau $q$ " prélevé sur l'excès du débit de la rivière en temps le cluc

Le rendement $\rho$ de l'opération est défini par l'expression :

$$
\rho=\frac{\Delta P}{q^{\prime \prime} h}=\frac{\dot{q}}{q^{\prime}}\left[\frac{\left(h+h^{\frac{3}{2}}-h^{\frac{3}{2}}\right.}{h V h+h}\right]
$$

Si nous posons $\frac{q^{\prime}}{q,}=\gamma$ et $\frac{h^{\prime}}{h}=n$, cette expression prend la forme :

$$
p=r\left(\left(1+n-\frac{1}{\sqrt{1+n}}\right)\right.
$$

Elle ne contient que des rapports, et demeure indépendante des valeurs alsolues $q, q^{\prime \prime}, h, h^{\prime}$. I e rendement sera donc le incme, quels que solent le délit et la hauteur le chute pour des rapports égaux de $\frac{i}{i}$ d'une part, de $\frac{h}{h}$ de
l'autre.

Telles sont les indications que la thérie a fournics à linventeur.

M. Herschel a trouvé dans son compteur Venturi, el dans le bief tareur de Holyoke, tous les instruments nécessaires

pour reconnaitre dans quelle mesure la théorie se retrouvait dans la pratique. Il a dirigré et suivi les éprouves avec l'habileté savante que ses travaux sur le Venturi ont mise en si haut relief.

Un compteur Venturi, placé sur la canalisation $\mathrm{E}$ reliant la turbine au compartiment ou fonctionnait l'apparcil aspirateur (fig. 1), faisait connaitre à chaque instant, et dans des limites d'exactitude inférieures à 2 pour 100 , le volume q' débité par la turbine.

Un second compteur, inséré sur le tuyau B d'alimen- 
tation du renforceur, donnait dans les mêmes conditions le volume $q$ ".

La pression $h$ était mesurée au moyen d'un manomètre placé en a sur la conduite B. Le vide $h^{\prime}$ était mesuré au moyen d'un manomètre $b$ à tubes recourbés, branché sur la " chambre de vide » $\mathrm{A}$.

Des vannes placées sur les canalisations E et B permeltaient de faire varier à volonté les débits $q$ ' et $q$ ".

Un flotteur $f$ indiquait le niveau de l'eau dans la chambre A.

La partie $\mathrm{C}$ du renforceur, produisant l'aspiration dans le compartiment $\mathrm{A}$, se compose d'un tuyau tronconique

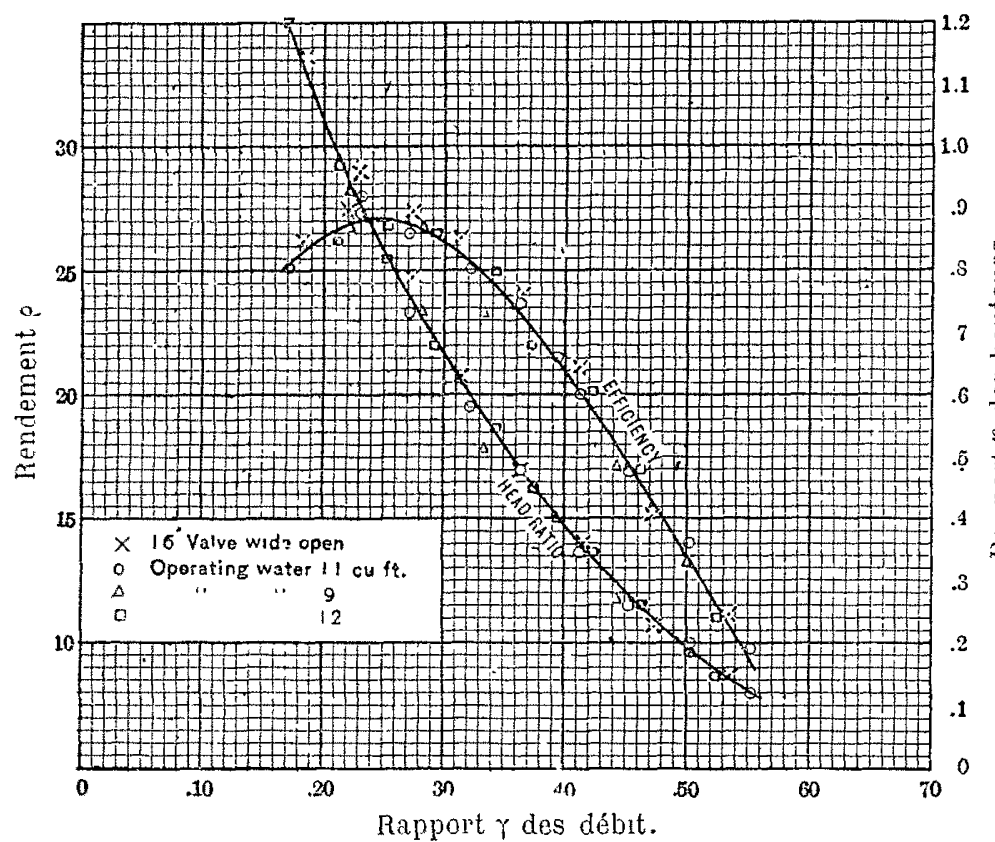

Fig. 2. - Graphiques de rendement du renforceur du type $F$. 16. Vulve wide open $=$ Valve de 16 inches ouverte en plein.

operaling water $11 \mathrm{cu} . \mathrm{ft} .=$ débit agissant : 11 preds cubes.

percé de trous. Il a fallu déterminer expérimentalement, et les dimensions à donner à ce tronc de cone, el la surface d’aspiration des ouvertures de succion. C'était là certainement une question fort délicate : les règles à suivre dans l'établissement de ces éléments du renforceur ne seront

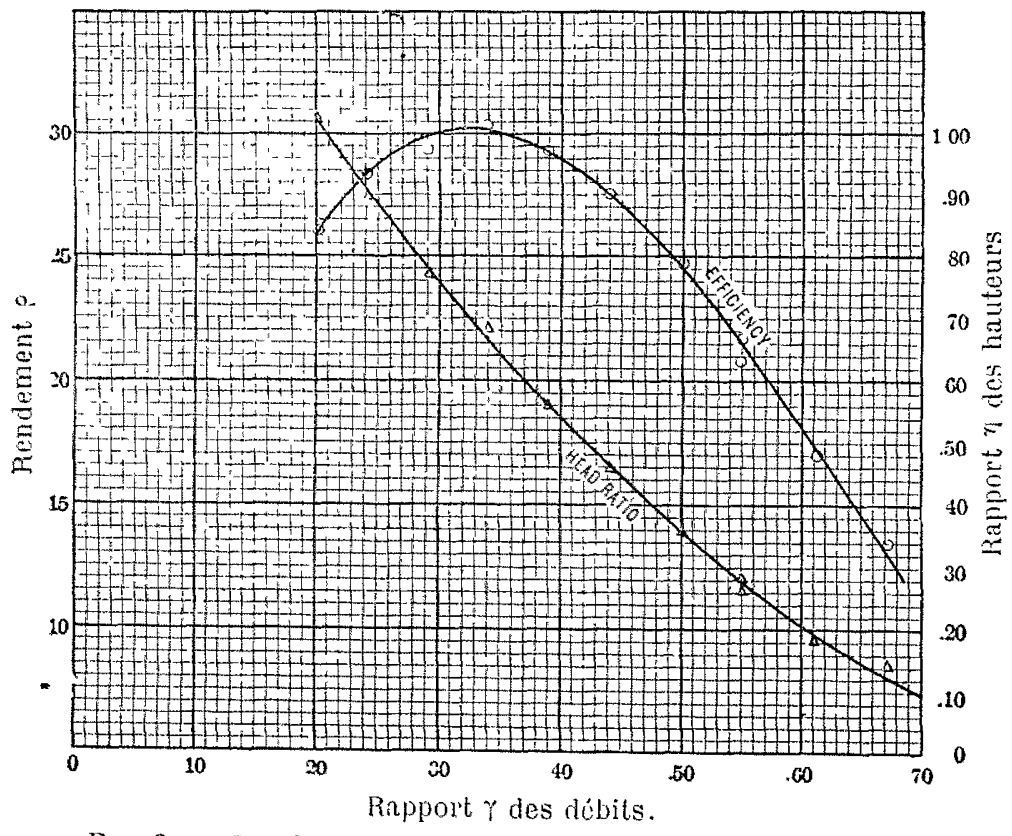

Fia. 3. - Graphiques de rendement du renforceur du type $B$.

sủrement définies quaprès des expériences faites sous des conditions de charge et de débit très différentes les unes dos autres. Six modèles onl été éprouvés, dont plusieur's furent rejetés parce qu'avec eux la relation qui unit le rapport des débits $\frac{q}{q}$, au rapport des pressions $\frac{h}{h}$ était troublée au point de les rendre inutilisables pour le renforceur de chute.

A la suite de ces essais, $M$. Herschel a retenu comme le meilleur le type de renforceur qu'il a désigné par la lettre $B$. Nous reproduisons ici les tableaux et courbes relatant les résultats obtenus avec les renforceurs des deux types, $B$ et $F$.

Tableau I. - Renforceur de chute. - Type F

\begin{tabular}{|c|c|c|c|c|c|c|c|c|c|c|}
\hline \multirow{2}{*}{ 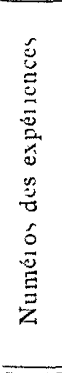 } & \multicolumn{2}{|c|}{$\begin{array}{l}\text { Hauteur } \\
\text { de l'eau } \\
\text { en metres }\end{array}$} & \multirow{2}{*}{ 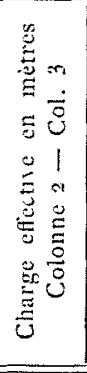 } & \multirow{2}{*}{ 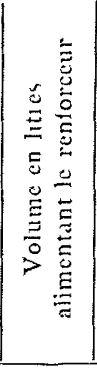 } & \multirow[b]{2}{*}{ 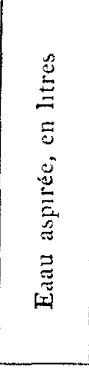 } & \multirow{2}{*}{ 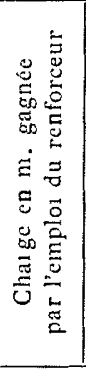 } & \multirow{2}{*}{ 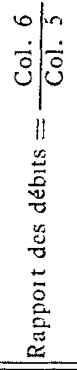 } & \multirow{2}{*}{ 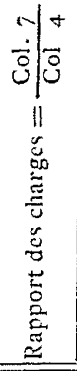 } & \multirow[b]{2}{*}{ 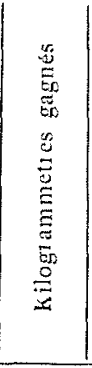 } & \multirow[b]{2}{*}{ 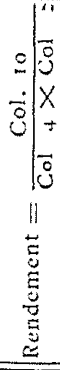 } \\
\hline & $\begin{array}{l}m \\
\Xi \\
\vdots \\
\Xi \\
\vdots \\
\vdots \\
\Xi \\
\vdots\end{array}$ & 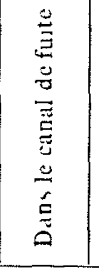 & & & & & & & & \\
\hline 1 & 2 & 3 & 4 & 5 & 6 & 7 & 8 & 9 & 10 & 11 \\
\hline $\begin{array}{l}1 \\
2 \\
3 \\
4 \\
5 \\
6 \\
7 \\
8\end{array}$ & $\begin{array}{l}28,941 \\
28,883 \\
28,782 \\
28,703 \\
28,615 \\
28,596 \\
28,444 \\
28,355\end{array}$ & $\begin{array}{l}24,997 \\
24,991 \\
24,994 \\
24,994 \\
24,994 \\
24,991 \\
24,991 \\
24,988\end{array}$ & $\begin{array}{l}3,943 \\
3,888 \\
3,788 \\
?, 708 \\
3,620 \\
3,534 \\
3,452 \\
3,367\end{array}$ & $\mid \begin{array}{l}322,33 \\
3: 9,19 \\
341 \\
350,63 \\
363,37 \\
373,56 \\
384,88 \\
395,36\end{array}$ & $\begin{array}{l}170,80 \\
156,65 \\
142,50 \\
138,35 \\
114,20 \\
100,05 \\
8 \pi, 90 \\
71,75\end{array}$ & $\begin{array}{l}0,588 \\
0,878 \\
1,378 \\
1,796 \\
2,287 \\
2,775 \\
3.303 \\
3,836\end{array}$ & $\begin{array}{l}0,53 \\
0,47 \\
0,41 \\
0,36 \\
0,31 \\
0,27 \\
0,22 \\
0,18\end{array}$ & $\begin{array}{l}0,15 \\
0,23 \\
0,36 \\
0,48 \\
0,63 \\
0,79 \\
096 \\
1,14\end{array}$ & $\mid \begin{array}{l}143,43 \\
194,86 \\
273,45 \\
312,40 \\
317,30 \\
363,32 \\
364,35 \\
346,92 \\
\end{array}$ & $\begin{array}{l}11,3 \\
15,2 \\
21,1 \\
24,0 \\
26,4 \\
27,5 \\
27,4 \\
26,1\end{array}$ \\
\hline $\begin{array}{r}9 \\
10 \\
11 \\
12 \\
13 \\
14 \\
15 \\
16\end{array}$ & $\mid \begin{array}{l}27,132 \\
27,367 \\
27,581 \\
27,861 \\
27,980 \\
28.307 \\
28,582 \\
28,727\end{array}$ & $\left|\begin{array}{r}21,952 \\
24,959 \\
24,96-1 \\
24,967 \\
24973 \\
24,979 \\
24,988 \\
21,991\end{array}\right|$ & $\begin{array}{l}2,180 \\
2,415 \\
2,616 \\
2,897 \\
3,007 \\
3,327 \\
3,544 \\
3,736\end{array}$ & $\left|\begin{array}{l}311,58 \\
311,58 \\
311,30 \\
312,71 \\
309,88 \\
311,30 \\
312,14 \\
310,73\end{array}\right|$ & $\begin{array}{r}71,77 \\
85,90 \\
110,05 \\
114,20 \\
128,35 \\
142,50 \\
156,65 \\
170,80\end{array}$ & $\begin{array}{l}2,006 \\
1,769 \\
1,525 \\
1,381 \\
1052 \\
0,872 \\
0,701 \\
0.457\end{array}$ & $\begin{array}{l}0,43 \\
0,27 \\
0,32 \\
0,36 \\
0,41 \\
0,45 \\
0,50 \\
0,55\end{array}$ & $\begin{array}{l}0,99 \\
0,73 \\
0,58 \\
0,18 \\
0,35 \\
0,26 \\
0,20 \\
0,12\end{array}$ & $\left|\begin{array}{l}184,85 \\
199,13 \\
203,84 \\
214,97 \\
187,44 \\
175,44 \\
156,46 \\
11357\end{array}\right|$ & $\begin{array}{r}27,2 \\
26.5 \\
25,0 \\
23,7 \\
20,1 \\
16,9 \\
11,1 \\
9,8\end{array}$ \\
\hline $\begin{array}{l}17 \\
18 \\
19 \\
20 \\
21 \\
22\end{array}$ & $\left|\begin{array}{l}27,239 \\
27,007 \\
26,861 \\
26,733 \\
26,489 \\
26,309\end{array}\right|$ & $\left|\begin{array}{l}24,949 \\
24,945 \\
24,939 \\
24,933 \\
24,924 \\
24,921\end{array}\right|$ & $\begin{array}{l}2,290 \\
2,101 \\
1,924 \\
1,799 \\
1,564 \\
1,387\end{array}$ & $\left|\begin{array}{l}254,98 \\
254,41 \\
253,85 \\
254,70 \\
254,40 \\
254,13\end{array}\right|$ & $\begin{array}{r}126,35 \\
114,20 \\
160,05 \\
85,90 \\
71,75 \\
57,60\end{array}$ & $\begin{array}{l}0,447 \\
0,564 \\
0,768 \\
0,921 \\
1,134 \\
1,267\end{array}$ & $\begin{array}{l}0,50 \\
0,44 \\
0,39 \\
0,33 \\
0,28 \\
0,22\end{array}$ & $\begin{array}{l}0,19 \\
0,27 \\
0,40 \\
0,51 \\
0,73 \\
0,93\end{array}$ & $\begin{array}{r}77,84 \\
91,20 \\
105,63 \\
106,83 \\
106,66 \\
94,84\end{array}$ & $\begin{array}{l}13,3 \\
17,1 \\
21,6 \\
23,3 \\
26,8 \\
26,8\end{array}$ \\
\hline $\begin{array}{l}23 \\
24 \\
25 \\
26 \\
27 \\
28 \\
29 \\
30 \\
32\end{array}$ & $\left|\begin{array}{l}27,245 \\
27,526 \\
27,736 \\
27,999 \\
28,227 \\
28,624 \\
28,767 \\
28,923 \\
29,042\end{array}\right|$ & $\left|\begin{array}{l}24,958 \\
24,961 \\
24,967 \\
24,973 \\
24,979 \\
24,991 \\
24,994 \\
24,997 \\
24,997\end{array}\right|$ & $\begin{array}{l}2,287 \\
2,562 \\
2,769 \\
3,025 \\
3,248 \\
3,632 \\
3,772 \\
3,9 \div 5 \\
4,044\end{array}$ & $\left|\begin{array}{l}341,01 \\
339,60 \\
339,31 \\
339,3 ! \\
337,05 \\
342, ; 1 \\
339,03 \\
337,33 \\
321,88\end{array}\right|$ & \begin{tabular}{|r|}
57,60 \\
71,75 \\
85,98 \\
100,05 \\
114,20 \\
$12 \cdot, 35$ \\
112,50 \\
156,65 \\
171,37
\end{tabular} & $\begin{array}{l}2,735 \\
2,491 \\
2,266 \\
2,058 \\
1,778 \\
1,616 \\
1,365 \\
1,027 \\
0,588\end{array}$ & $\begin{array}{l}0,17 \\
0,21 \\
0,25 \\
0,29 \\
0,34 \\
0,37 \\
0,42 \\
0,46 \\
0,52\end{array}$ & $\begin{array}{l}1,20 \\
0,97 \\
0,82 \\
0,68 \\
0,55 \\
045 \\
0,35 \\
0,26 \\
0,15\end{array}$ & $\left|\begin{array}{l}196,93 \\
228,34 \\
253,20 \\
272,10 \\
273,65 \\
283,84 \\
257,95 \\
226,62 \\
146,19\end{array}\right|$ & $\begin{array}{l}25,2 \\
26,3 \\
26,9 \\
26,5 \\
26,11 \\
22,1 \\
20,2 \\
17,1 \\
11,1\end{array}$ \\
\hline
\end{tabular}

Tableau II. - Renforceur de chute. - Type B

\begin{tabular}{|c|c|c|c|c|c|c|c|c|c|c|}
\hline 1 & 2 & 3 & 4 & 5 & 6 & 7 & 8 & 9 & 10 & 11 \\
\hline 1 & $292 \leqslant 3$ & 24,985 & 4,257 & 306,48 & 169,80 & 1,128 & 0,55 & 0,27 & 272,44 & 20,9 \\
\hline 2 & 29.310 & 24988 & 4,322 & 299,41 & 183,95 & 0,832 & 0,61 & 0,19 & $2,0.81$ & 17,1 \\
\hline 3 & 29,368 & 24991 & 4,376 & 244,32 & 197,81 & 0,010 & 0,67 & 0,11 & 174,32 & 13,5 \\
\hline 4 & 29,243 & 24,988 & 4,255 & 306,77 & 16980 & 0,183 & 0,55 & $0,2 x$ & 28479 & 21,8 \\
\hline 5 & 29,179 & 21,988 & 4,193 & 13,56 & 155,30 & 1,509 & 0,50 & 0,36 & 396,55 & 24.8 \\
\hline 6 & 29,087 & 24,985 & 4.102 & 322,05 & 141,50 & 1869 & 044 & 0,16 & 369.97 & 27,5 \\
\hline 7 & 21,014 & $24,9 \& 2$ & 4,032 & 329,41 & 127,35 & 2,247 & 039 & 0,56 & 389,0 & 29,3 \\
\hline 8 & 28,932 & $249 \div 6$ & 3,955 & 337,05 & 113,20 & 2,677 & $0,3 \mathfrak{k}$ & 0,68 & 404,91 & 30,4 \\
\hline 9 & 28.871 & 2490 & 3,894 & 34294 & 99,05 & 3,004 & 0,20 & 0,77 & 343,35 & 29,4 \\
\hline 10 & 28.788 & 24,973 & 3,815 & 350,36 & 84,90 & 3,431 & 0,21 & 0,90 & 379,72 & 28,4 \\
\hline 11 & 28,718 & 24,970 & 3,748 & 356,58 & 70,75 & 3,818 & 0,20 & 1,02 & 348,30 & 26,1 \\
\hline
\end{tabular}

L'examen de la courbe relative au renforceur $F$ démontre que, conformément aux indications de la formule théorique, le reudement ne varie pas quand les volumes débités sont différents, si toutefois le rapport $\frac{q^{\prime}}{q^{\prime \prime}}$ des débits ou le rapport des charges $\frac{h}{h}$ restent les mêmes.

Ce fait acquis, la courle a été, pour le renforceur $B$, simplement établie avec le plein débit de la turbine. 
Le lecteur verra aisément comment il est possible de déterminer quelle est la surcharge à rechercher dans un cas donné.

Soil $H-h$ la hauteur de chute perdue en période de crue, $h^{\prime}=n h$ la charge traditionnelle que l'on voudrait obtenir. in regard du chiffre représentant la valeur de $n$, on lira un rapport $\frac{q^{\prime}}{q^{\prime \prime}}=\gamma$; on calculera $q^{\prime}$ en fonction d'éléments comnus : $Q$ débit normal de la turbine, $H, h, q^{\prime}=Q \frac{\sqrt{h+h^{\prime}}}{\sqrt{H}}$ et l'on verra si le volume $q "=\frac{q}{\gamma}$, nécessaire pour produire dans la chambre de vide la dépression $h$, peut se trouver dans le débit total $W$ en grandes eaux, diminué du volume $q$ ' absorbé par la lurbine. C'est en vue des débits $q$ ' et $q$ "que l'on devra prévoir le renforceur.

on détermine ainsi le régime pour la période que nous supposons maxima en durée, ou en valeur absolue de la crue. Dans la période intermédiaire, on produira, avec un rendement moindre il est vrai, des hanteurs de chute additionnelles $h_{1}^{\prime}, h_{2}^{\prime}, h_{3}^{\prime}$, qui seront égales a $n_{1} h_{1}, n_{2} h_{2}$, $x_{3}, h_{3} \ldots, h_{1}, h_{2}, h_{3} \ldots$ décroissant, tandis que $n_{1}, n_{2}, n_{3} \ldots$ grandiront, parce qu'on fournira au renforceur des volumes d'eau $q^{\prime \prime}{ }_{1}, q^{\prime \prime}, q^{\prime \prime}{ }_{3} \ldots$ de plus en plus grands à mesure que la crue grandıra elle-mème.

Le maximum de rendement correspond, pour le renforceur de chute $B$, a un rapport de débit $\frac{q^{\prime}}{q^{\prime \prime}}=0,33$, et pour le renforceur du type $F$ à un rapport de 0,25. Dans le premier cas, le volume d'cau alimentant le renforceur devi"a être égal à trois fois le débit de la turbine; dans le second, à quatre fois. Dans l'un et dans l'autre, la hauteur de chute gannée sera importante, mais. en " pratique, dit M. Herschel, il semblera meilleur de prendre un moyen terme, et de ne pas rechercher la plus grande charge additionnelle "; c'est ainsi qu'il recommande, avec le renforceur du trpe $B$, d'adopter un rapport de charge de 50 pour 100 , et un rapport de débit de 0,42 , coriespondants à un rendement de 28 pour 100. Observons, avec l'auteur, quin accrossement de charge de 50 pour 100 produira un accroissement de puissance plus considérable, théor1quement égal à 1,30 .

Nous ajouterons que le volume $q$ ", dont on peut disposer pour alimenter le renforceur, n'est pas illimitó; diutre part, les dimensions qu'il est possible de donner au tronc de cóne appelé à débiter les volumes q' et $q$ " no peuvent croître indéfiniment; en beaucoup de cas, l'état des lieux leur imposera un maximum. Lne nécessité de ce genre avait conduit $\mathrm{M}$. Herschel à prévoir, dans son projet pont. l'usine de la Plaine, près de Genève, deux renforceurs par turbiue, et á donner à ceux-ci, pour section droite, une cllipse à grand axe horizontal; il arrivait ainsi à un moindre encombrement en hauteur.

L'inventeur attire très justement notre attention sur le fait que l'emplacement destiné au renforceur pouvant etre pris dans les fondements même d'une usine génératrice, linstallation en est peu coûteuse.

Plusieurs installations de cos appareils sont actuellement à l'étude en Amérique. Des licences pour leur construchon ont eté acquises en France (*), en Allemagne et en Suisse. Nous ne doutons pas que $M$. Ilerschel ne recueille prócieusement toutes les indications que lui fournira lou fonctionnement dans des conditions très différentes les unes des autres, et nous sommes certains qu'il en tirera des enseignements féconds. Comme il l'a toujours fail, il communiquera à ses collogrues des deux mondes le lésultat de ses précieux travaux, et acquerra un nonveau

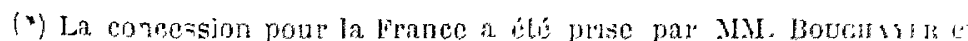
Vallet, conelructeurs à Grenoble.
}

titre à l'estime et la reconnaissance de tous les ingénieurs attachés à l'Hydraulique industrielle.

Remarque. - S'adressant à ses collègues de Ilarward, M. Ilerschel insistait sur l'énorme importance réservée à un laboratoire où seraient étudiés, d'une farcon continue et à une échelle suffisante, les nombreux problemes que comporte l'hydraulique. Il observait que plusieurs n'ont été résolus que par le fait du liasard, inettant aux prises avecl'un d'eux un homme compétent, et qu'en ces conditions le progrès devait nécessairement être lent et accidentel.

Nous sommes heureux de placer sous un patronage aussi autór'sé des observations analogues.

Si l'utilisation des cluutes de falble et moyenne hauteur présente encore des difficultés multiples, alors mêmequ'elleest depuis si longtemps pratiquée et que, par suite, les conditions favorables a ux. quelles MI Ilerschel faitallusion ont été bien des foís rćalisées, il

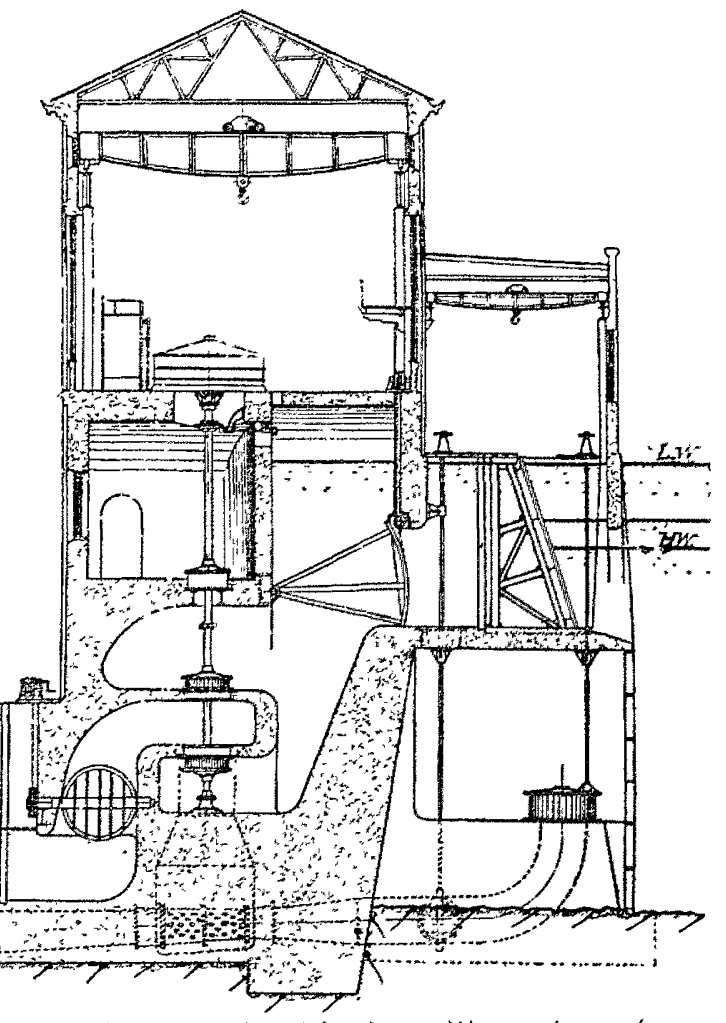

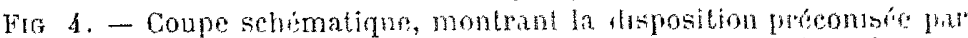
M. Hersehell, pour l'emplos de son renforcent de chute, lors du concours organisé par la ville de cineve nour laménagement de 1 usinot hydro-blectrique de La Planc (1).

e:t incontestable que des difficultes plus graves et plus nombreuses se rencontrent quamr incrénieurs ot constructeurs veulent asservir des chutes de grande hauteur.

Les règles, les formules auxquelles ils peuvent recourir. en confiance dans le premier cas n'offent plus, dans le second, une égale sécrité Des phénomònes interviennent qui démentent ce qui avait été reconnu suffisant ou rat, mais ne l'est en réalité que dans cel laines lunites de pussance ou de pression.

Isa nécessité d'éclairer certaines questions douleuses, ou au moins vagues, a été comprise lors du Congrès de la Iouille Blanche. A sa suite, une Commission, dite des turbines, a été instituée; dle poursuit ses travaux dont le prosramme comprend essnotielement is jangeage rapile ex exact dans les canalisations libre= ou sous pression, lo rendement et la légulation des turbines; cufin, los coups de bclier.

Grace à l'obligeance de sociétés industrielles. Ia Commission peut tenter un certain nombre dexperiences sur des installations existantes, mais on comprend aisement quelle ne peut le faire qu'avec une extrome discrution. 11 cu scrait autrement si un lahoratoire hydrauhune lui flait librement et constamment ouvert.

Le Ministere de l'Agriculture a charge deux ingeniours, que désignaient tout naturriliment à son choix leur hauto

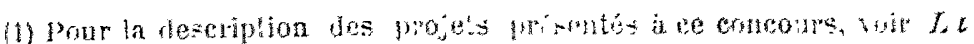
Houtlle Blancle de mars 1906. 
valeur technique et de remarquables travaux, MM. de la Brossc et Tavernier, d'étudier le régime complet des eaux dans les massifs montagneux de la France.

Cette marque d'ntérêt pour l'avenir des industries hydrauliques laisse espérer que l'Administration aiderait à la création d'un laboratoire où serait étudié l'ensemble des questions relatives à la meilleure utilisation de nos chutes d'eau

Mais il somblerait nécessaire que linitiative de cette fondation fùt price par les intéressés Ceux-ci sont nombreux, parmi les lecteur's de La Houille Blanche, et c'est à leur bienveillante attention que, loccasion s'oflrant, nous soumeltons ce projet.

Augustin BLANCHET, Ingénceur des Arts el Manufaclures.

La Houille Blanche. qu depuis sa fondation fall appel au concours ses lecteurs pour seconder la Commission des Essais de turbines hydraulluues, se joint à M. Blanchet pour unviter les hydrauhciens à réaliser la fondation qu'll préconise si justement.

X D.L.R.

\section{LA RICHESSE DU RHONE et son utilisation}

\author{
'Suile et (in)
}

Rhône navigable - Je suppose que la questıon du Rhòne " navigable n, qu sulfrat à absorber le temiss que vous jouvez miaccorder ce sonr. vous est famılère. Je ne la traiteral donc pas avec toute lampleur qu elle mérite.

Le ròle économique du Rhòne dans longaanısatıon geénérale des transports est tellement restreint par rapyort à ce quil levraut étre, que cest un sujet détonnement, on peut le drre, unversel. Jajouteras mème que cel étonnement se manifeste au loin plus vivement encore rue sur les bords du fleuve. Il est bien mpossible que les Lyonnars et les autres ruverans du Rhòne princupalement intéressés ne commencent pas à se blaser sur un sujet rebatlu jusqu'à la saliété saus quiil sorte de tant de discussions des résultats tangibles.

Ciest dans un rapport d'ensemble sur les roies navigables de France, récemment présenté à la chambre de Commerce lOorléans par M. Arnodin, que je trouve défins, de la façon la plus vive, le ròle que devraient jouer Lron et le Rhòne dans lorgamisalion des transports.

Falsant allusion aux projets suisses, dont je vous disais un mot en commencant. M. Arnodm ajoule que cependant " le noud navigable de l'Europe centrale est Lyon et non " pas Bâle; la grande voie internationale deau douce faisant. " sulte au canal martime de Suez pour gagner le Nord de " lEurope est : Marselle, Lyon, Paris, Rouen, le Hâvre ; " celle pour gagner l'Atlantique : Marseille, Lxon, Saint"Ftienne, Roanne, Orléans, Nantes, Saml-Nazaire et, plus " au sud, Marseille, Beaucaire, Toulouse, Bordeaux ".

Que faudrait-1l faire? Il faudrait, c'est toujours M. Arnodin qui parle, non pas en Lyonnais mais en Françaıs envisageant l'intérèt général du pays et s'occupant. ne l'oubliez pas, de l'onsemble des voies navigables de la France : "il faudrait, " premièrement, se hâter d"ouvrir le canal latéral du Rhône, " y compris le raccordement de Marseille au Rhòne, actuel"lement en construction : car le tronçon principal qui empê" che le corps entier dietre viabie est le Rhône sur lequel ne " peut sieffectuer malheureusement qu'une navigation pré" caire".

Sur cette questinn du Rhône navigable, vous savez que doux courants dopinon bien tranchés se sont manifestés. Les uns croient, avec M. Arnodin, quil niy a plus rien à faire du Rhòne actuel, du Rhòne amélioré, autant qu'il était possible de lespérer par les travaux si réussis de M. Girar-

don (1). A ceux qu: pensent ainsı, le développement insın. fiant du tonnage transporté par le Rhòne, fournit le melleur des arguments : l'argument de falt ( $(2)$. Les autres, sans nier aucunement la superiorité d'un canal sur un cours d'eau aussi impélueux que le Rhòne, sinquiètent surtoul des possibılıtés financières et voudraient qu'on cherchât à tırer un melleur parti de l'outil avant de le mettre au rebut. Ces derniers ne manquent pas, non plus, de bonnes raisons. Le Rhône, dépourvu de ports et de movens de trackons publics, n’est pas, en réalité, ouvert à la batellerıe générale. Le développement de son trafic est limité par la lenteur avec laquelle la seule Compagnı naviguant sur le Rhòne augmenta sa flotte et ses installations. D'autre part, la voie navigable Rhône-Saòne, artère unique de Saint-Louis à Ghalon, est isolée par la politıque de la Compagnie P.-L.-M. des sources de trafic qui ne sont pas situées sur des bords. Faute de racicordement avec les voies ferrées, elle ne dessert ni les bassins houillers de la région, nı les industrıes métallurgiques de Samt-Etienne, ni les industries de houll. blanche qui se multiplient dans les Alpes. Si J'on parvient à faire du Rhòne une vole réellement ollverte à tous, en y créant des ports publics raccordés, et des services publics de traction, on peut espérer que le trafic se développera sur le Rhòne comme il s'est développé sur les fleuves allemand: dont les conditions de navigabilité ne sont pas supérıeures.

Ces ldées opposées se sont heurlées avec éclat au Congrès des Chambres de Commerce qui s'est tenu à Lyon en 1899. sous la présidence de M. Isaac. Notre cher collègue a thù déployer les éminentes qualilés que vous lui connaissez, el qui font de lui le plus, achevé des Présidents, pour contenir les ardeurs des représentants du Midi soulevés contre le Rhône "navigable ", accusé de faire du tort au Rhône " agricole ". On a finalement confiél le soin de concilier ces vues contradictoires à l'Office des transports dont on déc1dait la création.

Voici sur quelles bases l'Office, s'acquittant de son mandat, s'est efforcé de faire concorder les efforts des Chambres de Commerce du Sud-Eist.

Aux partisans impatients du canal latéral, il a rappelé la grandeur de l'œuvre, le temps nécessaire pour dégager par des études complexes la meilleure solution, les délais plus longs encore indispensables pour mettre sur pied Jes combmaisons financières. Il est sage, en attendant, de ne pas abandonner un moyen de transport qui, tel qu'il est, rend des services directs et indirects. Plus on développera Je trafle du Rhône en améliorant les raccordements, créant des ports publics et des services publics de traction, et plus on aura une base financière solide pour l'établissement d'une voie navigable plus parfaite.

Aux esprits enclins à s'attarder dans le statu quo, l'office des transports a signalé les circonstances nouvelles qui donnaient aux études à entreprendre un intérêt particulier. On ne saurait étudier avec tropı de soin ni trop à l'avance, si coûteuses et si difficiles à réaliser cu'elles paraissent, des

1) D’après $M$. Laffitte l'Elbe et l'Oder ont offert un tirant d'eau de Im45 respectivement pendant 195 et 127 jours.

D'après les plus récents rapports de $M$ Girardon, le tirant d'eau de 1 m60 était oblenu sur le Rhône pendant 165 jours avant les travaux, et pendant 327 jours après les travaux.

(2) Le développement du tonnage du Rhône est caractérisé par les chiffres qui sulvent:

$\begin{array}{cc}\text { Années } & \begin{array}{c}\text { Tonnage moyen du Rhòe } \\ \text { ramené s̀ la distance } \\ \text { entlère }\end{array} \\ \text { (entre Lyon e t Arles) } \\ 1883 & 218318 \mathrm{~T} \\ 1903 & 310708 \\ 1906 & 249.081\end{array}$

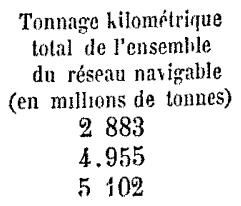

Trafic des deux lignes P.-L -M riveraines du Rhône entre Lyon et Arles en 1904 (Tonnage moyen à distance entière).

Ligne de rive gauche...... .................. . . . . . . . . . — de rive drotte, Givors à Lavoulte. . . . . . . . . . . . . 